\title{
Protective effect of chorioamnionitis on the development of bronchopulmonary dysplasia triggered by postnatal systemic inflammation in neonatal rats
}

\author{
Chang Won Choi ${ }^{1,2}$, Juyoung Lee ${ }^{2}$, Joo Youn $\mathrm{Oh}^{3}$, Seung Hyun Lee ${ }^{4}$, Hyun Ju Lee ${ }^{5}$ and Beyong II Kim ${ }^{1,2}$
}

BACKGROUND: Prenatal or postnatal systemic inflammation can contribute to the development of bronchopulmonary dysplasia (BPD). We investigated whether prenatal intra-amniotic (i.a.) inflammation or early postnatal systemic inflammation can induce BPD in a rat model.

METHODS: One microgram of lipopolysaccharide (LPS) or vehicle was injected into the amniotic sacs $2 \mathrm{~d}$ before delivery (E20). After birth, $0.25 \mathrm{mg} / \mathrm{kg}$ of LPS or vehicle was injected into the peritoneum of pups on postnatal day (P)1, P3, and P5. On P7 and P14, peripheral blood (PB), bronchoalveolar lavage fluid (BALF), and lung tissue were obtained and analyzed.

RESULTS: Postnatal i.p. injections of LPS significantly increased neutrophil counts in PB and BALF on P7 and P14. Similarly, proinflammatory cytokine and angiogenic factor transcript levels were increased in the lung by i.p. LPS on P7. Alveolar and pulmonary vascular development was markedly disrupted by i.p. LPS on P14. However, pretreatment with i.a. LPS significantly negated the detrimental effects of postnatal i.p. LPS on PB and BALF neutrophil counts and on lung proinflammatory cytokine expression and histopathological changes.

CONCLUSION: Exposure to early postnatal systemic LPS induces BPD, an arrest in alveolarization, in neonatal rats. Preceding exposure to i.a. LPS protects the lungs against BPD triggered by postnatal systemic inflammation.

$\mathbf{T}$ he incidence of bronchopulmonary dysplasia (BPD) has not changed over the past decade despite enormous improvements in preterm newborn care (1). Meanwhile, the clinical manifestation and pathology of BPD have evolved from "old BPD" to the so-called "new BPD" as neonatal medicine has advanced and clinical practices have become more sophisticated (2). Antenatal corticosteroids, pulmonary surfactants, and noninvasive ventilation technologies have allowed for the use of lower concentrations of oxygen and less mechanical ventilation in clinical practice for very preterm infants compared with previous treatment strategies (3). Furthermore, compared to presurfactant era, current BPD occurs in more immature infants born at 22 to $26 \mathrm{wk}$ ' gestation whose lungs are in the late canalicular or early saccular phase of lung development (4). These changes in clinical practice and epidemiology subsequently led to changes in the clinical manifestation and histopathology of BPD $(5,6)$. "New BPD" exhibits less airway epithelial damage, airway smooth muscle hyperplasia, and fibroproliferative changes than "old BPD." Therefore, alveolar and pulmonary vascular hypoplasia has replaced the classic histopathological findings of "old BPD" and has become a hallmark of "new BPD" (6).

Inflammation plays a central role in the pathogenesis of BPD (7). Inflammation can be evoked by infectious organisms, oxidative stress, or mechanical ventilation (8). Oxidative stress from using a high oxygen concentration and baro/volutrauma with mechanical ventilation are, to date, considered the most crucial pathogenetic factors for BPD $(9,10)$. However, because less oxygen and noninvasive ventilation are becoming the standard of care for very preterm infants, sources of inflammation other than oxidative stress and mechanical injury such as chorioamnionitis and postnatal infection are becoming increasingly important as pathogenetic factors for BPD.

Classic rodent models of BPD have been established by exposure to hyperoxia and/or mechanical ventilation $(11,12)$. However, to simulate "new BPD," as is found in this postsurfactant era, a new animal model in which BPD is induced by means other than hyperoxia or mechanical injury is required. In this study, we tested whether BPD can be induced in rats by the administration of prenatal intra-amniotic (i.a.) lipopolysaccharide (LPS), postnatal i.p. LPS, or both without hyperoxia exposure (Figure 1). The interaction between i.a. LPS and i.p. LPS in developing BPD and inflammation was also investigated.

\section{RESULTS}

\section{Survival Rate}

The survival patterns in each group until postnatal day $(\mathrm{P}) 7$ are presented in Figure 2. Few fetal deaths (1/86) occurred in the i.a. vehicle-treated groups. However, the i.a. LPStreated groups showed a modest fetal death rate of $12.2 \%$

\footnotetext{
'Department of Pediatrics, Seoul National University College of Medicine, Seoul, Republic of Korea; ${ }^{2}$ Department of Pediatrics, Seoul National University Bundang Hospital, Seongnam, Republic of Korea; ${ }^{3}$ Department of Ophthalmology, Seoul National University College of Medicine, Seoul, Republic of Korea; ${ }^{4}$ Department of Pediatrics, Wonkwang University School of Medicine, Iksan, Republic of Korea; ${ }^{5}$ Department of Pediatrics, Hanyang University College of Medicine, Seoul, Republic of Korea. Correspondence: Beyong II Kim (beyil@snu.ac.kr)

Received 20 April 2015; accepted 8 August 2015; advance online publication 2 December 2015. doi:10.1038/pr.2015.224
} 


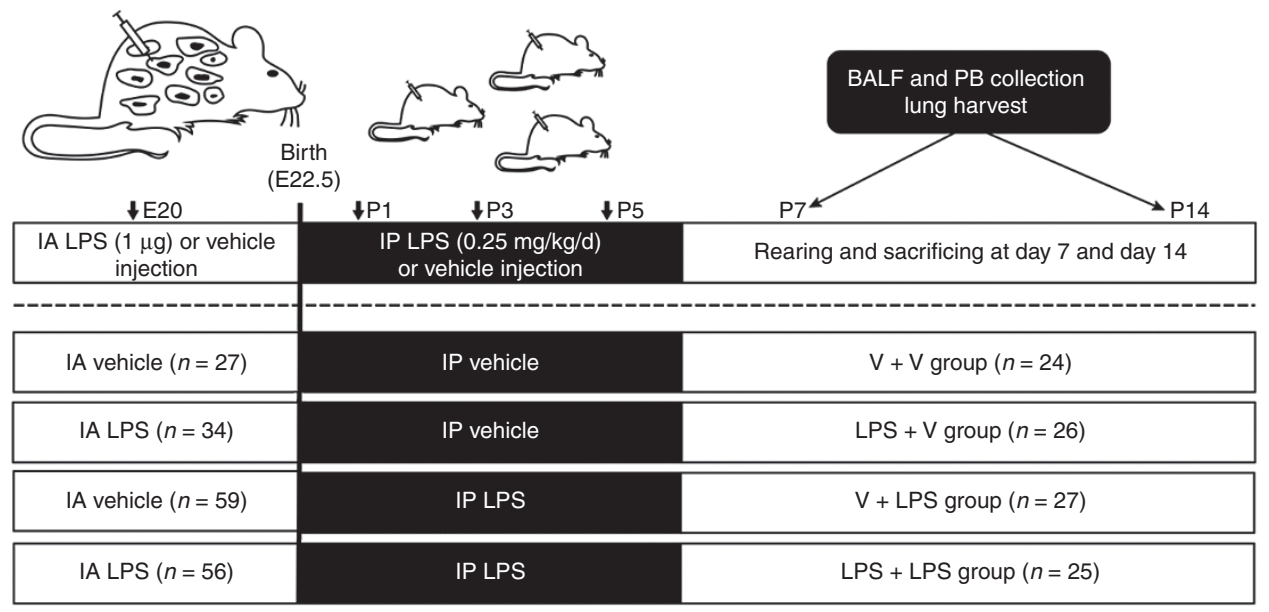

Figure 1. Schematic outline of the experimental protocol. Intra-amniotic LPS (1.0 $\mu \mathrm{g} / \mathrm{sac})$ or vehicle was administered on E20. Postnatal i.p. LPS (0.25 mg/ $\mathrm{kg} / \mathrm{d}$ ) or vehicle was administered on P1, P3, and P5. BALF and PB collection and lung harvesting were performed on P7 and P14. BALF, bronchoalveolar lavage fluid; IA, intra-amniotic; IP, intraperitoneal; PB, peripheral blood; V, vehicle.

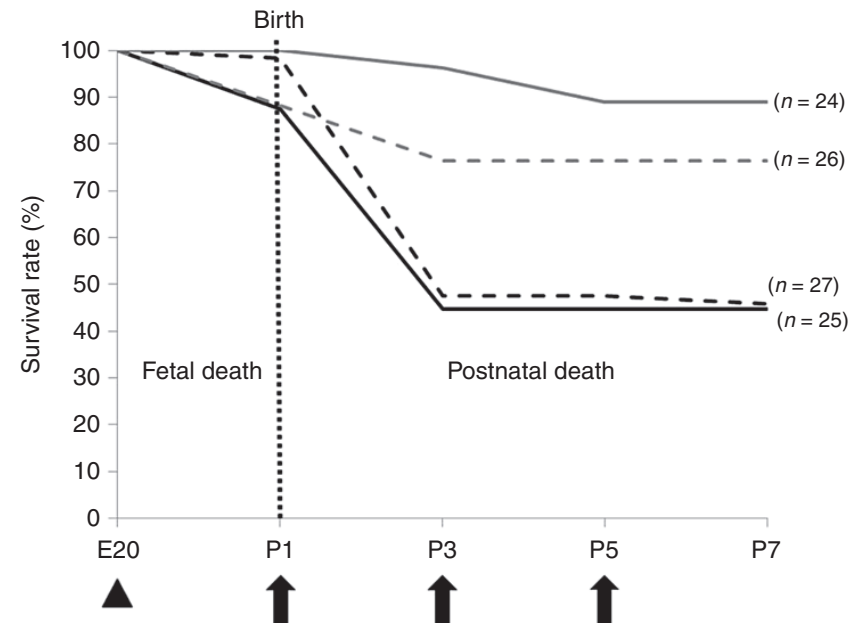

Figure 2. Survival rates in the experimental groups. Fetal death mostly occurred in the intra-amniotic (i.a.) LPS-treated groups. After birth, most of the deaths occurred in the i.p. LPS-treated groups. Nearly half of the rats in the i.p. LPS-treated groups died during the first several days after birth. In all groups, no further mortality occurred beyond P7. Gray solid line, V+V; gray dashed line, LPS+V; black dashed line, V+LPS; black solid line, LPS+LPS. Group abbreviation: V+V, i.a. vehicle followed by i.p. vehicle-treated group; LPS+V, i.a. LPS followed by i.p. vehicle-treated group; V+LPS, i.a. vehicle followed by i.p. LPS-treated group; LPS+LPS, i.a. LPS followed by i.p. LPS-treated group. The arrowhead indicates i.a. LPS $(1.0 \mu \mathrm{g} / \mathrm{sac})$, and the arrows indicate postnatal i.p. LPS $(0.25 \mathrm{mg} / \mathrm{kg} / \mathrm{d})$ administrations.

(11/90). After birth, 51.7\% (30/58) of the animals in the i.a. vehicle $(\mathrm{V})$ followed by i.p. LPS-treated groups (V+LPS) and $49.0 \%(24 / 49)$ of the animals in the i.a. LPS followed by i.p. LPS-treated groups (LPS+LPS) died after the first i.p. LPS injection. In contrast, $88.9 \%(24 / 27)$ of the animals in the i.a. vehicle followed by i.p. vehicle-treated groups $(\mathrm{V}+\mathrm{V})$ and $86.7 \%(26 / 30)$ of the animals in the i.a. LPS followed by i.p. vehicle-treated groups (LPS+V) survived the first 7 $\mathrm{d}$ after birth. In all groups, no further mortality occurred beyond P7.
White Blood Cell and Neutrophil Counts in Bronchoalveolar Lavage Fluid and Peripheral Blood

Postnatal i.p. LPS significantly increased the total white blood cell (WBC) counts in the bronchoalveolar lavage fluid (BALF) at P7 and P14 (Figure 3a). The total BALF neutrophil counts on P7 were significantly higher in the V+LPS group compared with the other groups (Figure $3 \mathbf{b}, \mathbf{c}$ ). The total BALF neutrophil counts were markedly decreased in all groups on P14 compared with those at P7. However, the V+LPS group had a significantly higher number of BALF neutrophils than the $\mathrm{V}+\mathrm{V}$ group. Similar findings were observed with the total WBC or neutrophil counts in peripheral blood (PB) on P7 (Figure 3d,e). Notably, the $\mathrm{V}+\mathrm{V}$ group had the highest total PB WBC and neutrophil counts on P14 among the groups.

\section{Alveolar Development}

The i.p. LPS-treated groups showed large and simple distal air spaces, the hallmark of disrupted alveolarization, on P7. The alveolarization proceeded on P14, as indicated by small and complex distal air spaces in all groups except the V+LPS group. In the V+LPS group, distal airspaces remained large and simple on P14, similar to their appearance on P7, indicating an arrest in alveolarization (Figure 4a). Morphometric assessment was consistent with these microscopic findings. The mean cord length $\left(\mathrm{L}_{\mathrm{m}}\right)$, which is an indicator of the average alveolar size, was significantly greater in the i.p. LPStreated groups than in the i.p. vehicle-treated groups on P7. The $\mathrm{L}_{\mathrm{m}}$ was decreased on P14 as the lungs developed in all groups, but the $\mathrm{V}+\mathrm{LPS}$ group had the greatest $\mathrm{L}_{\mathrm{m}}$ among the groups (Figure $4 b$ ). In contrast, the alveolar surface area $\left(S_{A}\right)$ was significantly smaller in the V+LPS group than in the i.p. vehicle-treated groups on P7. The $\mathrm{S}_{\mathrm{A}}$ was increased on P14 as the lungs developed in all groups. However, the i.p. LPStreated groups had a smaller $S_{A}$ than the i.p. vehicle-treated groups (Figure 4c). The alveolar wall thickness $\left(\mathrm{W}_{\mathrm{T}}\right)$ was significantly thinner in the i.p. LPS-treated groups compared with the $\mathrm{V}+\mathrm{V}$ group on $\mathrm{P} 7$. The $\mathrm{W}_{\mathrm{T}}$ was thinned on $\mathrm{P} 14$ as the 
a

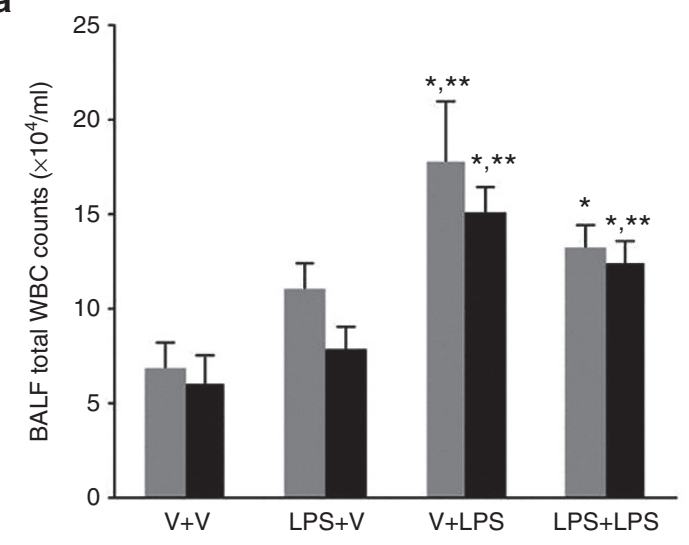

b

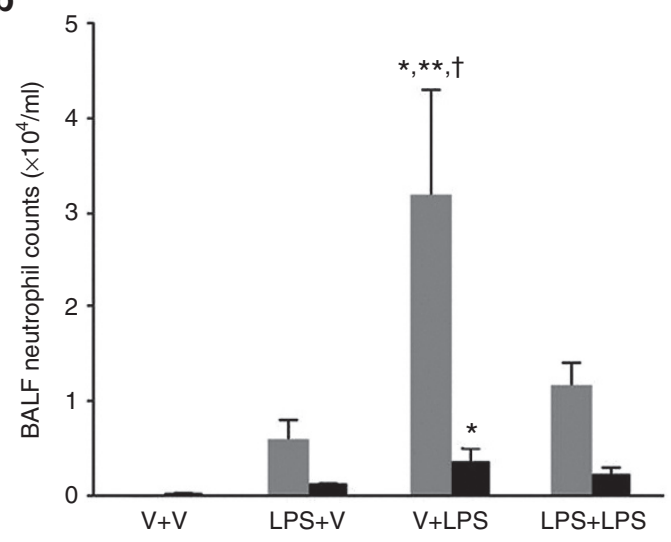

C

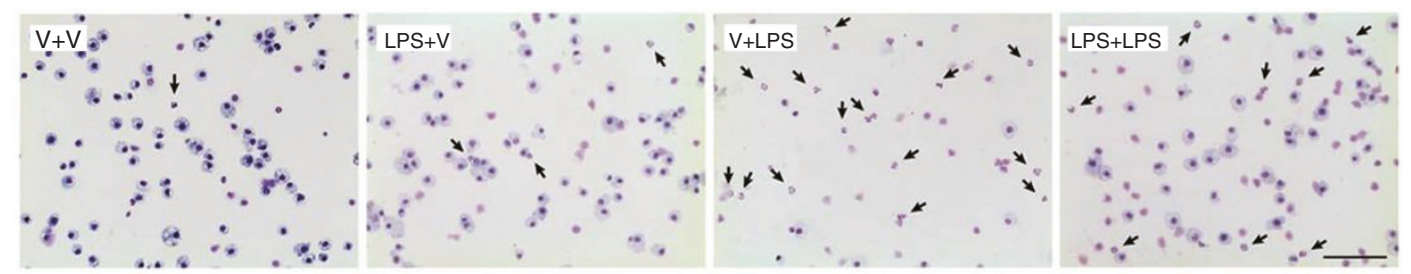

d
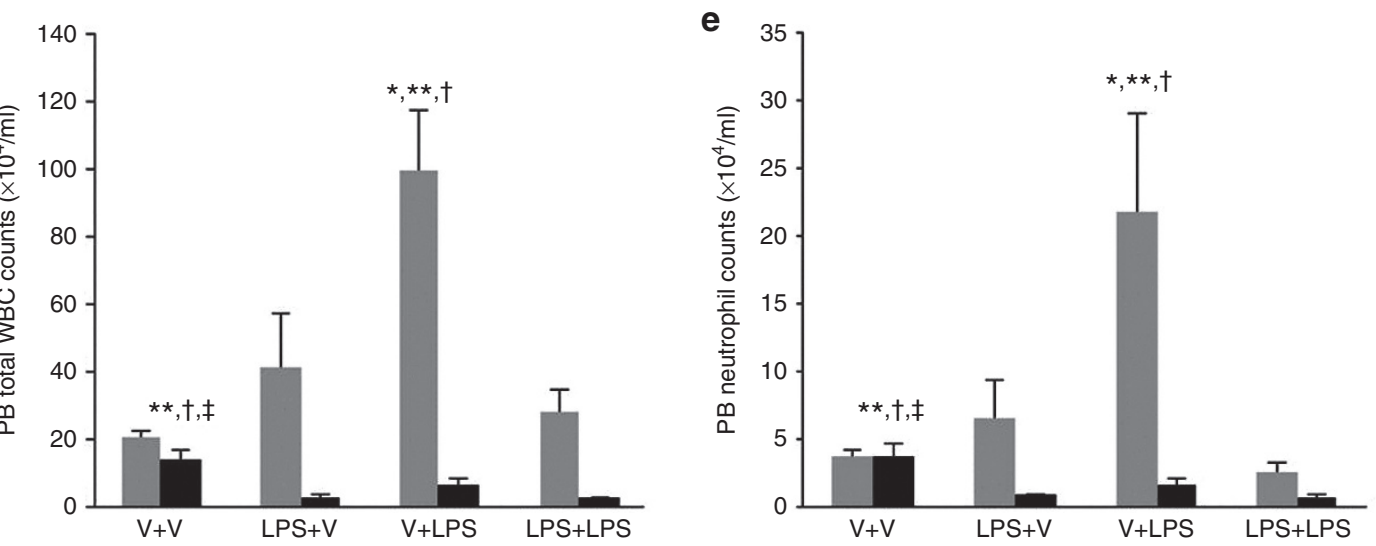

Figure 3. Bronchoalveolar lavage fluid and peripheral blood white blood cell and neutrophil counts. (a, b, d, e) Gray bars denote P7 and black bars P14. (a) The total BALF WBC counts were higher in the i.p. LPS-treated groups than in the i.p. vehicle-treated groups on P7 and P14. (b) The V+LPS group had higher BALF neutrophil counts than in other groups on P7 and in the V+V group on P14. (c) Representative light microscopic images of the cytospin BALF samples on P7. A marked increase in the number of neutrophils in the BALF cytospin sample from the V+LPS group is observed compared with the other groups. The arrows indicate neutrophils. Diff-Quik (Sysmex, Kobe, Japan) stained. Original magnification $\times 400$. The bar indicates $50 \mu \mathrm{m}$. (d, e) The total PB WBC and neutrophil counts were highest in the V+LPS group on P7. On P14, the total WBC and neutrophil counts in PB were reduced compared with $P 7$, and the $V+V$ group had the highest number of total WBC and neutrophil counts in PB. Group abbreviation: $V+V$, i.a. vehicle followed by i.p. vehicletreated group; LPS+V, i.a. LPS followed by i.p. vehicle-treated group; V+LPS, i.a. vehicle followed by i.p. LPS-treated group; LPS+LPS, i.a. LPS followed by i.p. LPS-treated group. The data are the mean $\pm \mathrm{SEM}\left(N=5-6\right.$ in each group). ${ }^{*} P<0.05 \mathrm{vs}$. $\mathrm{V}+\mathrm{V},{ }^{* *} P<0.05 \mathrm{vs}$. LPS $+\mathrm{V},{ }^{+} P<0.05 \mathrm{vs}$. LPS $+\mathrm{LPS},{ }^{\ddagger} P<0.05 \mathrm{vs}$. V+LPS. $B A L F$, bronchoalveolar lavage fluid; $P B$, peripheral blood; $V$, vehicle; $W B C$, white blood cell.

lungs developed in all groups except the V+LPS group. The $\mathrm{W}_{\mathrm{T}}$ of the V+LPS group changed little between P7 and P14 and was the thickest on P14 (Figure 4d).

\section{Pulmonary Vascular Development}

A considerably lower number and density of blood vessels were observed in the i.p. LPS-treated groups compared with the i.p. vehicle-treated groups on P7 and P14 (Figure 5a). The vessel density but not vessel number was significantly lower in the V+LPS group than in the LPS+LPS group. On P14, the number and density of the vessels remained significantly low in the i.p. LPS-treated groups compared with the i.p. vehicle-treated groups. The number and density of the vessels of the V+LPS group were significantly lower compared with the LPS+LPS group (Figure $5 b, c)$.

\section{Real-Time Quantitative RT-PCR}

Proinflammatory cytokine (TNF- $\alpha$, IL-1 $\beta$, IL-6, IL-12A) and angiogenic factor (VEGF, hypoxia-inducible factor (HIF-1 $\alpha)$ ) transcript levels in the lung tissues were significantly higher in the $\mathrm{V}+\mathrm{LPS}$ group than in the $\mathrm{V}+\mathrm{V}$ group on P7 (Figure 6a). Similarly, TNF- $\alpha$, IL-12A, and VEGF transcript levels on P14 were significantly higher in the $\mathrm{V}+\mathrm{LPS}$ group than in the $\mathrm{V}+\mathrm{V}$ group (Figure 6b). However, no differences in proinflammatory cytokine and angiogenic factor levels between the $\mathrm{V}+\mathrm{V}$ and LPS+LPS groups were found on P7 and P14 (Figure 6a,b). 
a
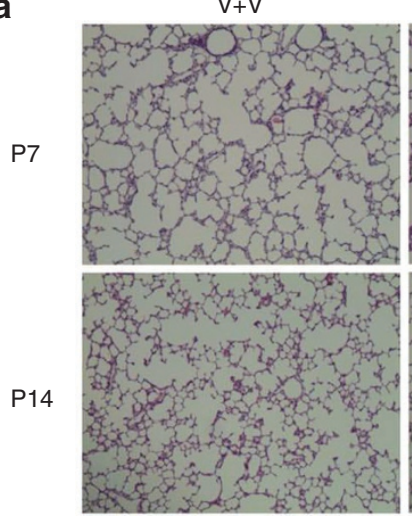

b

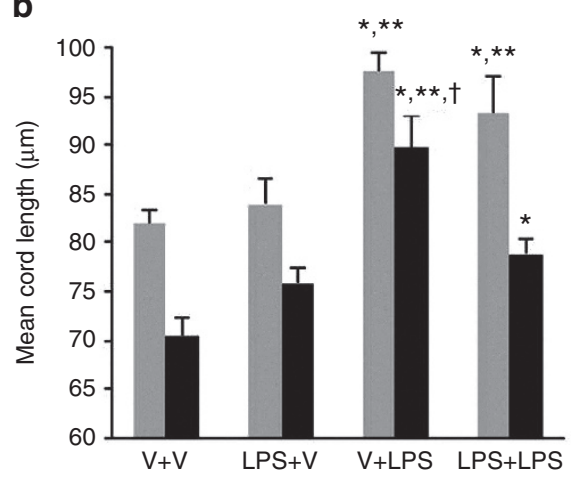

LPS+V

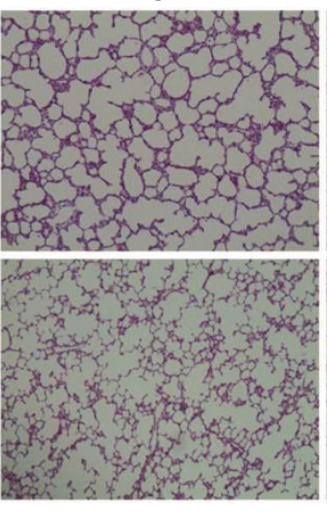

C

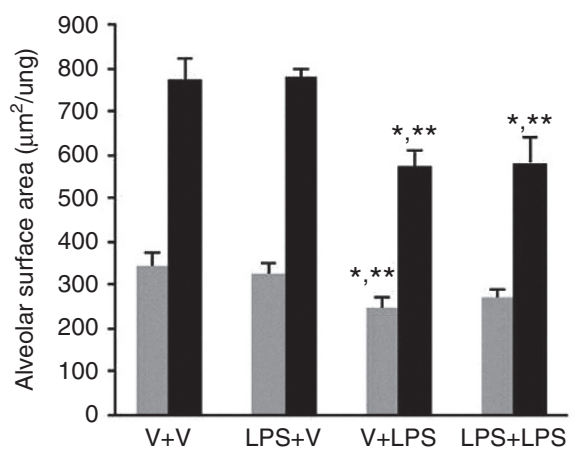

V+LPS

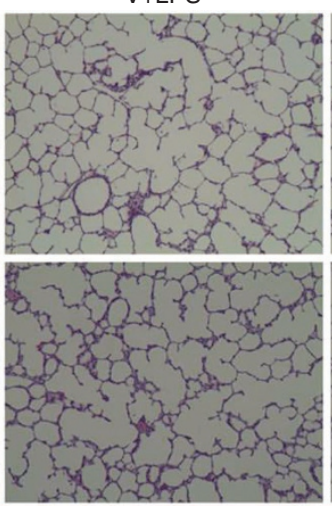

LPS+LPS

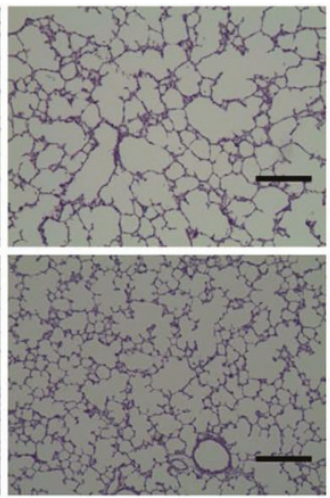

d

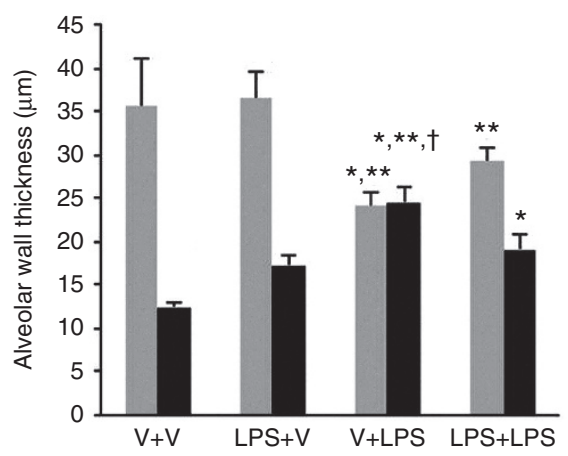

Figure 4. Alveolar development and morphometric data. (a) Representative light microscopic images of the rat lungs on P7 and P14. Apparently thinned septal walls were observed in the V+LPS group compared with other groups on P7, and markedly large and simple airspaces were observed in the V+LPS group compared with other groups on P14. Hematoxylin and eosin stained; original magnification $\times 100$. Bars, $200 \mu$ m. (b-d) Gray bars denote P7 and black bars P14. (b) The mean cord length $\left(\mathrm{L}_{m}\right)$, indicating the average alveolar size, was greater in the i.p. LPS-treated groups than in the i.p. vehicletreated groups on P7. The $L_{m}$ was decreased on P14 as the lungs developed in all groups, but V+LPS group had the highest $L_{m}$ among the groups. (c) The alveolar surface area $\left(S_{A}\right)$ was smaller in the $V+L P S$ group than in the i.p. vehicle-treated groups on P7. The $S_{A}$ increased on P14 as the lungs developed in all groups, but the i.p. LPS-treated groups had a smaller $S_{A}$ than the i.p. vehicle-treated groups. (d) The alveolar wall thickness ( $W_{T}$ ) was thinner in the i.p. LPS-treated groups than in the $\mathrm{V}+\mathrm{V}$ group on $\mathrm{P7}$. The $\mathrm{W}_{\mathrm{T}}$ was thinned on P14 as the lungs developed in all groups except the $\mathrm{V}+\mathrm{LPS}$ group, and the $\mathrm{V}+\mathrm{LPS}$ group had the thickest $W_{T}$ on P14. Group abbreviation: $V+V$, i.a. vehicle followed by i.p. vehicle-treated group; LPS+V, i.a. LPS followed by i.p. vehicletreated group; $V+L P S$, i.a. vehicle followed by i.p. LPS-treated group; LPS+LPS, i.a. LPS followed by i.p. LPS-treated group. The data are the mean \pm SEM ( $N=6-7$ in each group). ${ }^{*} P<0.05$ vs. $V+V,{ }^{* *} P<0.05$ vs. LPS $+V^{+} P<0.05$ vs. LPS+LPS. , vehicle.

\section{DISCUSSION}

A number of animal models of neonatal lung injury or BPD with an emphasis on postnatal inflammation have been reported (13). Furthermore, combination models using two or more different stimuli to induce lung injury have been reported, including our previous studies (14-17). However, few animal models of BPD have combined prenatal and postnatal inflammation. We combined prenatal i.a. LPS and postnatal i.p. LPS as stimuli to induce lung injury in this study. Prenatal i.a. LPS was administered on E20, which corresponds to the late canalicular stage of rat lung development, and postnatal i.p. LPS was administered on P1, P3, and P5, which correspond to the saccular and early alveolar stages (18). In this fetal and neonatal rat model, early postnatal i.p. LPS significantly inhibited lung development, whereas prenatal i.a. LPS alone did not affect lung development. These findings suggest a direct detrimental effect of early postnatal systemic inflammation on lung development. The degree of impaired alveolar and pulmonary vascular development paralleled the degree of pulmonary and systemic inflammation.
Based on the previous study (14), we used $1.0 \mu \mathrm{g}$ of LPS for the i.a. injection. For the postnatal i.p. injection, we used $0.25 \mathrm{mg} / \mathrm{kg}$ LPS. Although the route of administration differed, $1.0 \mu \mathrm{g}$ i.a. LPS is comparable to $0.4-0.5 \mathrm{mg} / \mathrm{kg}$ because the mean fetal weight of the rats on E20 was 2.0-2.5 g (19). Therefore, we used a 1.6-2.0 times lower dose of LPS for the postnatal i.p. LPS injection than that for the i.a. injection. This lower dose was used because in our preliminary study, the postnatal i.p. injection of $0.5 \mathrm{mg} / \mathrm{kg}$ LPS on P1 killed all rat pups within $24 \mathrm{~h}$.

Intra-amniotic LPS affects the fetal innate immune system differently than systemically administered LPS (20). Intra-amniotic LPS directly contacts the respiratory tract, gastrointestinal tract, and skin and stimulates the local innate immune system. However, systemically introduced LPS induces an overwhelming systemic inflammatory response syndrome leading to high mortality (21). Consistent with these reports, our findings suggest that i.a. and i.p. LPS administration have differential effects on lung development and mortality. In our study, both BALF neutrophil counts and lung proinflammatory cytokine levels 
a

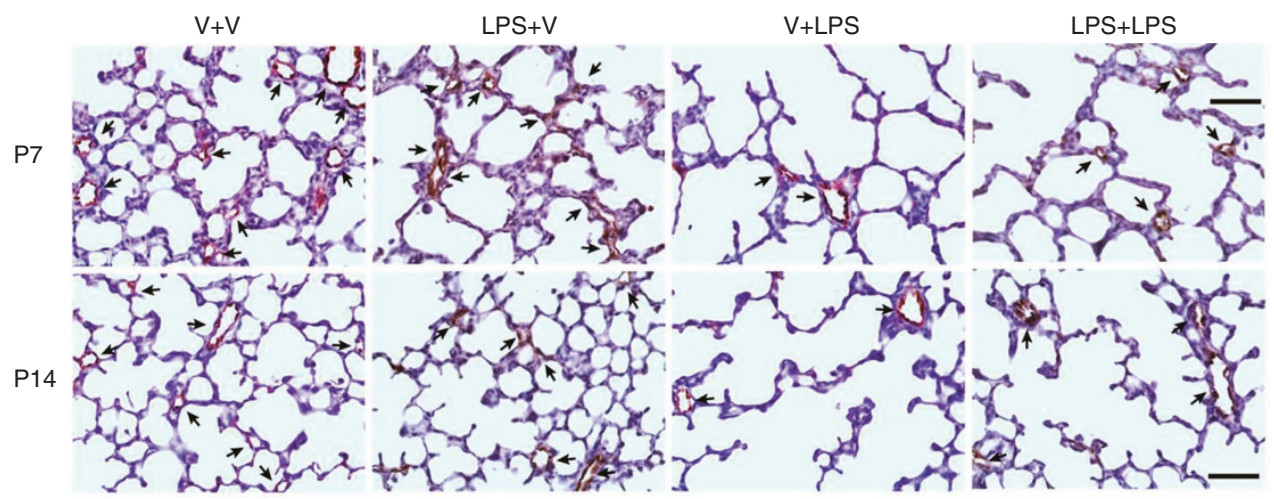

b

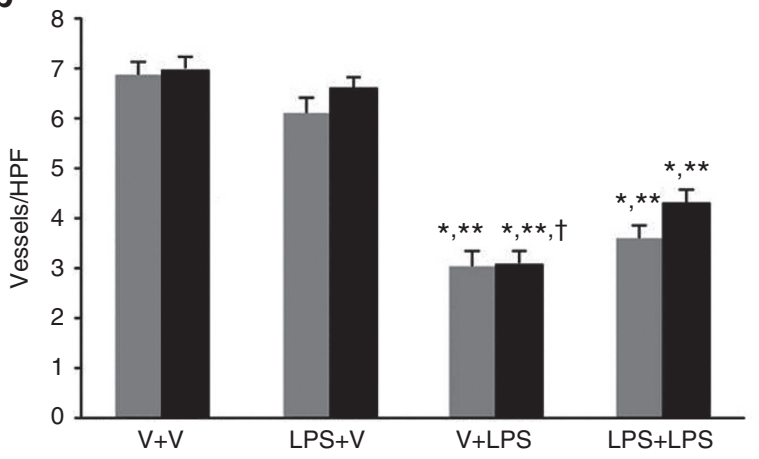

C

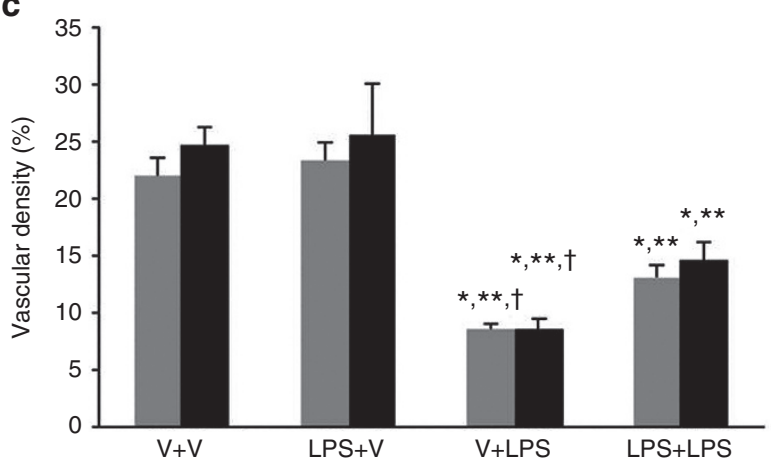

Figure 5. Pulmonary vascular development. (a) Representative light microscopic images of von Willebrand factor (vWF) immunohistochemistry of rat lung sections on P7 and P14. Apparently fewer blood vessels and decreased vWF staining were observed in the V+LPS group compared with the other groups on P7 and P14. The arrows indicate vessels. vWF staining was visualized with a DAB reaction (brown color). Light hematoxylin and eosin staining was used as a counterstain. Original magnification, $\times 400$. Bars $=50 \mu \mathrm{m}$. (b, c) Gray bars denote P7 and black bars P14. (b) The number of blood vessels per HPF was significantly lower in the i.p. LPS-treated groups than in the i.p. vehicle-treated groups on P7 and P14. On P14, the V+LPS group had a significantly lower number of vessels per HPF than the LPS+LPS group. (c) The vascular density, which was expressed as the ratio of the vWF-positive area to the total area of the lung parenchyma, was significantly lower in the i.p. LPS-treated groups than in the i.p. vehicle-treated groups on P7 and P14. The V+LPS group had a significantly lower vascular density than the LPS+LPS group on P7 and P14. Group abbreviation:V+V, i.a. vehicle followed by i.p. vehicletreated group; LPS+V, i.a. LPS followed by i.p. vehicle-treated group; V+LPS, i.a. vehicle followed by i.p. LPS-treated group; LPS+LPS, i.a. LPS followed by i.p. LPS-treated group. The data are the mean \pm SEM ( $N=6-7$ in each group). ${ }^{*} P<0.05$ vs. $V+V,{ }^{* *} P<0.05$ vs. LPS $+V ;{ }^{\dagger} P<0.05$ vs. LPS + LPS. HPF, high-powered field; $\mathrm{V}$, vehicle. DAB, diaminobenzidine.

were similar with or without i.a. LPS administration, suggesting that i.a. LPS alone might not induce inflammatory changes. Alternatively, the inflammatory changes might have returned to control levels on P7 after an initial increase after i.a. LPS administration. Regardless of the induction of inflammation, i.a. LPS did not alter lung development, whereas i.p. LPS did. Further studies will be necessary to determine whether the significant disruption of lung development by postnatal i.p. LPS was due to severe inflammation or to the timing of the LPS injection.

Another interesting finding of our study is that i.a. LPS attenuated the detrimental effects of subsequent postnatal i.p. LPS on systemic and pulmonary inflammation. Additionally, the i.a. LPS significantly reduced the inhibitory effects of i.p. LPS on alveolar and pulmonary vascular development. These findings suggest that the i.a. inflammation may protect the process of lung development from the postnatal systemic inflammation. These beneficial effects of i.a. LPS pretreatment might be related to endotoxin tolerance. Endotoxin tolerance has been demonstrated in preterm animals in the context of chorioamnionitis $(20,22)$. Repeated exposure to i.a. LPS decreased the expression of proinflammatory cytokines and toll-like receptor 4 in the lungs compared with a single i.a. LPS exposure in preterm lambs (20). Repeated i.a. LPS exposure has also been shown to induce cross-tolerance to other toll-like receptor agonists (23). Furthermore, a single i.a. LPS exposure led to lung remodeling similar to that found in BPD in the preterm lamb fetuses, whereas repeated i.a. LPS exposure did not $(24,25)$. Our and others' findings suggest that i.a. LPS may protect preterm lungs against subsequent inflammatory stimuli by inducing immune tolerance.

Structural maturation of the lungs includes a reduction of the alveolar septal thickness and restructuring of the double capillary system to a single capillary system (18). In this study, i.p. LPS led to alveolar wall thinning on P7, which was precocious compared with the control group. This precocious lung maturation was followed by an arrest in alveolar and pulmonary vascular development. These findings suggest that early postnatal systemic inflammation may induce precocious lung maturation at the expense of developmental potential.

Proinflammatory cytokine and angiogenic factor mRNA expression was markedly induced on P7 in the lungs of rats exposed to i.p. LPS without preceding i.a. LPS exposure. The 

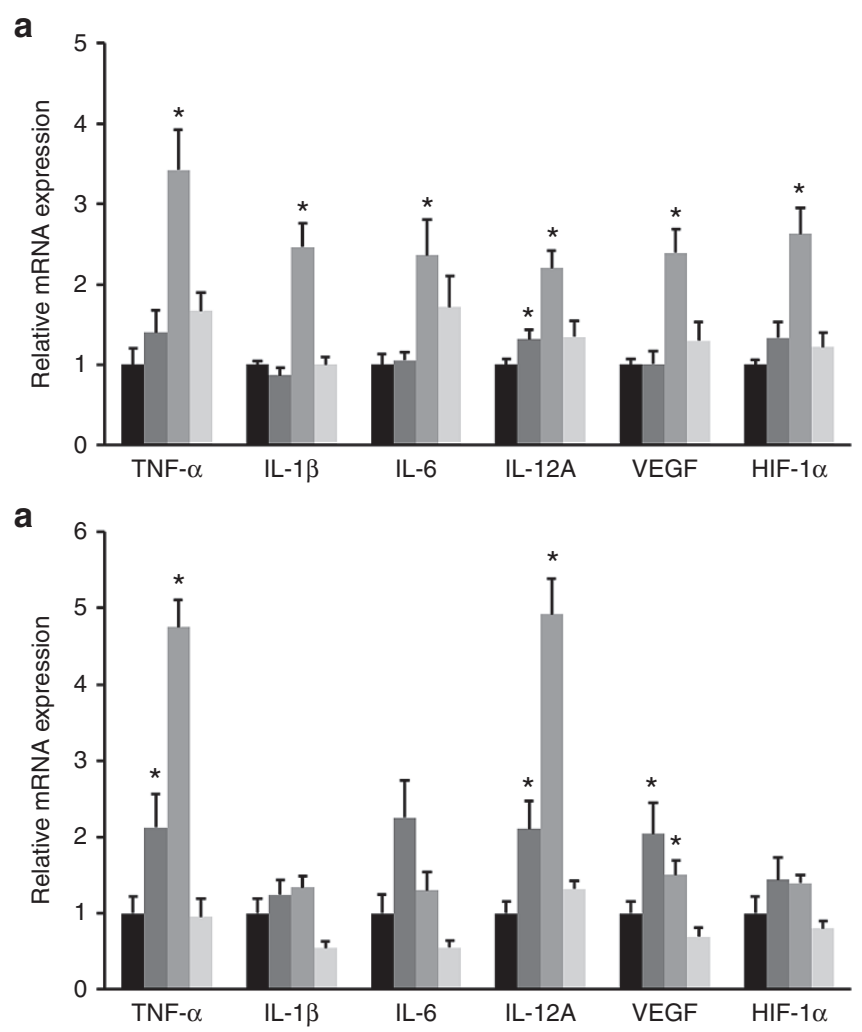

Figure 6. Inflammatory cytokine and angiogenic factor mRNA expression in lung tissue. The transcript levels of proinflammatory cytokines (TNF- $\alpha$, IL-1 $\beta$, IL-6, IL-12A) and angiogenic factors (VEGF, HIF-1 $\alpha$ ) on (a) P7 (b) and P14. The transcript levels of TNF- $\alpha$, IL-1 $\beta$, IL- 6, IL-12A, VEGF, and HIF- $1 \alpha$ in the lung tissue were significantly higher in the $\mathrm{V}+\mathrm{LPS}$ group than in the $\mathrm{V}+\mathrm{V}$ group on $\mathrm{P7}$. The transcript levels of TNF- $\alpha$, IL-12A, VEGF remained significantly higher in the $\mathrm{V}+\mathrm{LPS}$ group than in the $\mathrm{V}+\mathrm{V}$ group on $\mathrm{P} 14$. The mRNA expression of each group is presented as the relative value to that of the $\mathrm{V}+\mathrm{V}$ group. Black bars, $\mathrm{V}+\mathrm{V}$; dark gray bars, LPS+V; gray bars, $\mathrm{V}+\mathrm{LPS}$; light gray bars, LPS+LPS. Group abbreviation: $\mathrm{V}+\mathrm{V}$, i.a. vehicle followed by i.p. vehicle-treated group; LPS+V, i.a. LPS followed by i.p. vehicle-treated group; V+LPS, i.a. vehicle followed by i.p. LPS-treated group; LPS+LPS, i.a. LPS followed by i.p. LPS-treated group. The data are the mean \pm SEM ( $N=6-7$ in each group). ${ }^{*} P<0.05$ vs. $V+V$. HIF, hypoxia-inducible factor.

increase of proinflammatory cytokines by i.p. LPS was markedly attenuated by i.a. LPS. The protective effect of i.a. LPS was also notable by the proinflammatory cytokine levels found in our study. The increased proinflammatory cytokines and angiogenic factors may be involved in altered lung development. Inflammation-induced nuclear factor $\kappa \mathrm{B}$ activation impairs branching morphogenesis in the developing lung (26); however, little is known regarding the exact mechanisms by which a variety of proinflammatory cytokines and angiogenic factors alter the normal alveolar and pulmonary vascular development, either alone or in concert. Although angiogenic factor (VEGF, HIF-1 $\alpha)$ mRNA expression was increased in the lungs, pulmonary vascular development was markedly inhibited. Thus, the increased mRNA expression of these angiogenic factors may be a consequence of a reparative or compensatory response to counteract inflammation or of a direct effect of LPS (27).

Previous studies have reported that early- or late-onset sepsis increases the risk of BPD in human preterm infants
$(28,29)$. We here demonstrated that early postnatal systemic inflammation induces alveolar and pulmonary vascular developmental arrest, which is a hallmark of new BPD, in a neonatal rat model. Additionally, previous studies observed that preterm infants exposed to histological chorioamnionitis were protected against BPD (30). Our findings showed attenuated inflammation and less disruption of lung development by i.a. LPS administration. Therefore, our results provide experimental evidence for their clinical observations.

In this study, a rat model was used, which implies the need of further studies in larger animals that are anatomically and physiologically similar to human preterm infants. Although a partial protection against adverse effects of postnatal i.v. LPS that was endowed by prenatal i.a. LPS has been demonstrated in a preterm lamb model (31), there have been few larger animal models that show both the individual and combined effects of prenatal and postnatal inflammation on lung development. Additionally, experiments using inflammatory stimuli other than LPS would help validate our findings because human preterm infants are exposed to many complex inflammatory stimuli besides LPS in utero or after birth. However, a full assessment of alveolar development was possible using the neonatal rat because alveolarization occurs from P4 to P14 in the rat (18).

Our rat model of BPD induced by early postnatal i.p. LPS morphologically resembles new BPD of human preterm infants. Therefore, this model can be used to explore the mechanism how various inflammatory stimuli alter normal lung development and to search for novel therapies for BPD that target inflammation.

\section{METHODS}

\section{Animal Experiments}

The animal experimental protocol was approved by the Seoul National University Bundang Hospital Institutional Animal Care and Use Committee (BA1201-096/003-01). On E20 (term 22.5 d), pregnant Sprague-Dawley rats were anesthetized by isoflurane inhalation. After making a midline abdominal incision, $1 \mu \mathrm{g}$ of LPS (Escherichia coli 0111:B4; Chemicon International, Temecula, CA) dissolved in $0.1 \mathrm{ml}$ of saline or the same volume of vehicle (saline) was injected into the amniotic sacs under direct visualization. Each pregnant rat received either LPS or vehicle. After recovery, pups were spontaneously delivered $2-2.5 \mathrm{~d}$ after the injections. At 10-12 h after birth, pups from each treatment group were randomly re-assigned to dams from the same treatment group to balance the litter sizes. The rat pups were i.p. injected with either $0.25 \mathrm{mg} / \mathrm{kg}$ of LPS dissolved in $0.1 \mathrm{ml}$ of saline or the same volume of vehicle on P1, P3, and P5 to encompass the saccular and early alveolar stages of lung development in the rat (18). This experimental protocol led to four treatment groups: (i) i.a. vehicle followed by i.p. vehicle-treated group $(\mathrm{V}+\mathrm{V})$; (ii) i.a. LPS followed by i.p. vehicle-treated group (LPS+V); (iii) i.a. vehicle followed by i.p. LPStreated group (V+LPS); and (iv) i.a. LPS followed by i.p. LPS-treated group (LPS+LPS). Throughout the experiment, the rat dams reared pups that were allocated to a single group only. A total of 176 fetal rats from 13 pregnant rats were used in the experiment. A schematic outline of the experimental protocol is presented in Figure 1.

Bronchoalveolar Lavage Fluid and PB Collection and Cell Counts On P7 and P14, BALF and PB were collected from the rat pups. Five to six rat pups per group were used for BALF collection at each time point. The methods for BALF collection and cytospin preparation was described previously (32). The cytospin samples were stained with DiffQuik (Sysmex, Kobe, Japan) to allow the identification of different cell types. To obtain differential counts, $200-300$ cells were manually counted 
on each glass slide at $\times 200$ magnification. The number of each WBC type was calculated as the percentage of each WBC multiplied by the total number of WBCs in the BALF. For BALF collection, a different set of animals from those used for histological analysis was used to avoid possible interference of the BALF procedure with the histological analysis. PB was directly drawn from the left ventricle under anesthesia immediately before opening the chest wall for lung harvest on P7 and P14. Six to seven rat pups per group were used to collect $\mathrm{PB}$ at each time point. The total WBC count of collected PB was performed with the Hemavet 950FS analyzer (Drew Scientific, Dallas, TX). A differential cell count was manually performed on each $\mathrm{PB}$ smear slide at $\times 400$ magnification using the same method as for BALF.

\section{Tissue Preparation and Immunohistochemistry}

On P7 and P14, rat pups were anesthetized, and their lungs were harvested and prepared for immunohistochemistry and histological analyses, as previously described $(14,33)$. For immunohistochemical evaluation of von Willebrand factor (vWF), the deparaffinized lung sections were incubated with polyclonal rabbit antihuman vWF antibody (DakoCytomation, Glostrup, Denmark) and a biotin-labeled donkey antimouse secondary antibody (Jackson ImmunoResearch Laboratories, West Grove, PA). Immune complexes were visualized using diaminobenzidine (Vector Laboratories, Burlingame, CA). Light hematoxylin and eosin was applied as a counterstain.

Evaluations for Alveolar and Pulmonary Vascular Development Four random, nonoverlapping fields per animal in two distal lung sections were captured at $\times 100$ magnification to assess alveolar development. The extent of alveolarization was objectively assessed using the $\mathrm{L}_{\mathrm{m}}$ and $\mathrm{S}_{\mathrm{A}}$, as previously described $(14,33)$. To measure the $\mathrm{W}_{\mathrm{T}}$, four random, nonoverlapping high-powered fields (HPFs) per animal in two distal lung sections were captured. The $\mathrm{W}_{\mathrm{T}}$ was measured using scale in random five areas on each HPF. A total of $20 \mathrm{~W}_{\mathrm{T}}$ measurements from five random areas on four random HPFs were performed for each animal. For pulmonary vascular development assessment, images of vWF-stained slides were captured at $\times 400$ magnification. The number of vWF-positive vessels $(<100 \mu \mathrm{m}$ in size $)$ per HPF was counted. The vascular density was expressed as the ratio of the vWFpositive area to the total area of lung parenchyma. Color thresholding of the gray-black diaminobenzidine staining was performed using the differential interference contrast image analysis function of Image-Pro Plus software (Media Cybernetics, Rockville, MD). At least five counts from five fields were performed for each animal, and the fields were randomly chosen in areas not containing large airways or vessels. Six to seven rat pups were examined per group.

\section{Real-Time Quantitative RT-PCR}

Lung tissues were lysed in RNA isolation reagent (RNA-Bee; TelTest, Friendswood, TX) and sonicated with an ultrasound sonicator (Ultrasonic Processor; Cole Parmer Instruments, Vernon Hills, IL). Total RNA was extracted using an RNeasy Mini kit (Qiagen, Valencia, CA) and quantified using a NanoDrop spectrophotometer (Thermo Fisher Scientific, Waltham, MA). Equal amounts of RNA from each sample were used to synthesize first-strand cDNA by reverse transcription (High Capacity RNA-to-cDNA Kit; Applied Biosystems/Life Technologies, Carlsbad, CA). Real-time PCR amplification was performed using TaqMan Universal PCR Master Mix (Applied Biosystems/ Life Technologies). Rat glyceraldehyde-3-phosphate dehydrogenase was used for gene expression normalization. The PCR probe sets for rat glyceraldehyde-3-phosphate dehydrogenase, TNF- $\alpha$, IL-1 $\beta$, IL-6, IL-12A, VEGF, and HIF-1 $\alpha$ were purchased from Applied Biosystems (TaqMan Gene Expression Assay kits). Six to seven rat pups were examined per group.

\section{Statistical Analyses}

The data are expressed as the mean \pm SEM. Comparisons were performed by Kruskal-Wallis ANOVA, and post-hoc differences were assessed by the Mann-Whitney $U$-test. $P$ values $<0.05$ were considered significant.

\section{STATEMENT OF FINANCIAL SUPPORT}

This study was supported by the National Research Foundation of Korea (NRF) Grant funded by the Korean Government (MOE) (2010-0021644), the Seoul National University Bundang Hospital Research Fund (grant no. 02-2013-118), and a grant of the Korean Health Technology R\&D Project funded by the Ministry of Health \& Welfare, Republic of Korea (A112023).

\section{REFERENCES}

1. Smith VC, Zupancic JA, McCormick MC, et al. Trends in severe bronchopulmonary dysplasia rates between 1994 and 2002. J Pediatr 2005;146: 469-73.

2. Jobe AH. The new BPD: an arrest of lung development. Pediatr Res 1999;46:641-3.

3. Rojas MA, Gonzalez A, Bancalari E, Claure N, Poole C, Silva-Neto G. Changing trends in the epidemiology and pathogenesis of neonatal chronic lung disease. J Pediatr 1995;126:605-10.

4. Van Marter LJ. Epidemiology of bronchopulmonary dysplasia. In: Abman SH, ed. Bronchopulmonary Dysplasia. New York: Informa Healthcare, 2010:223-66.

5. Charafeddine L, D’Angio CT, Phelps DL. Atypical chronic lung disease patterns in neonates. Pediatrics 1999;103:759-65.

6. Husain AN, Siddiqui NH, Stocker JT. Pathology of arrested acinar development in postsurfactant bronchopulmonary dysplasia. Hum Pathol 1998;29:710-7.

7. Speer CP. Inflammation and bronchopulmonary dysplasia: a continuing story. Semin Fetal Neonatal Med 2006;11:354-62.

8. Wright CJ, Kirpalani H. Targeting inflammation to prevent bronchopulmonary dysplasia: can new insights be translated into therapies? Pediatrics 2011;128:111-26.

9. Askie LM, Henderson-Smart DJ, Irwig L, Simpson JM. Oxygen-saturation targets and outcomes in extremely preterm infants. N Engl J Med 2003;349:959-67.

10. Mokres LM, Parai K, Hilgendorff A, et al. Prolonged mechanical ventilation with air induces apoptosis and causes failure of alveolar septation and angiogenesis in lungs of newborn mice. Am J Physiol Lung Cell Mol Physiol 2010;298:L23-35.

11. Berger J, Bhandari V. Animal models of bronchopulmonary dysplasia. The term mouse models. Am J Physiol Lung Cell Mol Physiol 2014;307: L936-47.

12. O’Reilly M, Thébaud B. Animal models of bronchopulmonary dysplasia. The term rat models. Am J Physiol Lung Cell Mol Physiol 2014;307:L948-58.

13. Bhandari V. Postnatal inflammation in the pathogenesis of bronchopulmonary dysplasia. Birth Defects Res A Clin Mol Teratol 2014;100:189-201.

14. Choi CW, Kim BI, Hong JS, Kim EK, Kim HS, Choi JH. Bronchopulmonary dysplasia in a rat model induced by intra-amniotic inflammation and postnatal hyperoxia: morphometric aspects. Pediatr Res 2009;65:323-7.

15. Wilson TC, Bachurski CJ, Ikegami M, Jobe AH, Kallapur SG. Pulmonary and systemic induction of SAA3 after ventilation and endotoxin in preterm lambs. Pediatr Res 2005;58:1204-9.

16. Kroon AA, Wang J, Huang Z, Cao L, Kuliszewski M, Post M. Inflammatory response to oxygen and endotoxin in newborn rat lung ventilated with low tidal volume. Pediatr Res 2010;68:63-9.

17. Trummer-Menzi E, Gremlich S, Schittny JC, et al. Evolution of gene expression changes in newborn rats after mechanical ventilation with reversible intubation. Pediatr Pulmonol 2012;47:1204-14.

18. Schittny JC, Burri PH. Development and growth of the lung. In: Fishman AP, Elias JA, Fishman JA, Grippi MA, Senior RM, Pack AI, eds. Fishman's Pulmonary Diseases and Disorders. 4th edn. New York: McGraw-Hill, 2008:91-114.

19. Romero A, Villamayor F, Grau MT, Sacristán A, Ortiz JA. Relationship between fetal weight and litter size in rats: application to reproductive toxicology studies. Reprod Toxicol 1992;6:453-6.

20. Kallapur SG, Jobe AH, Ball MK, et al. Pulmonary and systemic endotoxin tolerance in preterm fetal sheep exposed to chorioamnionitis. J Immunol 2007;179:8491-9.

21. Jobe AH, Newnham JP, Willet KE, et al. Endotoxin-induced lung maturation in preterm lambs is not mediated by cortisol. Am J Respir Crit Care Med 2000;162:1656-61.

22. Kramer BW, Ikegami M, Moss TJ, Nitsos I, Newnham JP, Jobe AH. Endotoxin-induced chorioamnionitis modulates innate immunity of monocytes in preterm sheep. Am J Respir Crit Care Med 2005;171:73-7. 


\section{Articles $\mid$ Choietal.}

23. Kramer BW, Kallapur SG, Moss TJ, Nitsos I, Newnham JP, Jobe AH. Intraamniotic LPS modulation of TLR signaling in lung and blood monocytes of fetal sheep. Innate Immun 2009;15:101-7.

24. Willet KE, Jobe AH, Ikegami M, Newnham J, Brennan S, Sly PD. Antenatal endotoxin and glucocorticoid effects on lung morphometry in preterm lambs. Pediatr Res 2000;48:782-8.

25. Kallapur SG, Nitsos I, Moss TJ, et al. Chronic endotoxin exposure does not cause sustained structural abnormalities in the fetal sheep lungs. Am J Physiol Lung Cell Mol Physiol 2005;288:L966-74.

26. Benjamin JT, Carver BJ, Plosa EJ, et al. NF-kappaB activation limits airway branching through inhibition of Sp1-mediated fibroblast growth factor-10 expression. J Immunol 2010;185:4896-903.

27. Pollet I, Opina CJ, Zimmerman C, Leong KG, Wong F, Karsan A. Bacterial lipopolysaccharide directly induces angiogenesis through TRAF6mediated activation of NF-kappaB and c-Jun N-terminal kinase. Blood 2003;102:1740-2.

28. Stoll BJ, Hansen N, Fanaroff AA, et al. Changes in pathogens causing early-onset sepsis in very-low-birth-weight infants. N Engl J Med 2002;347:240-7.

29. Stoll BJ, Hansen N, Fanaroff AA, et al. Late-onset sepsis in very low birth weight neonates: the experience of the NICHD Neonatal Research Network. Pediatrics 2002;110:285-91.

30. Lahra MM, Beeby PJ, Jeffery HE. Intrauterine inflammation, neonatal sepsis, and chronic lung disease: a 13-year hospital cohort study. Pediatrics 2009;123:1314-9.
31. Gisslen T, Hillman NH, Musk GC, et al. Repeated exposure to intraamniotic LPS partially protects against adverse effects of intravenous LPS in preterm lambs. Innate Immun 2014;20:214-24.

32. Lee HJ, Lee YJ, Jo HS, et al. Double exposure to intra-amniotic lipopolysaccharide and maternal betamethasone induces sustained increase of neutrophils in the lungs and disrupts alveolarization in newborn rats. J Perinat Med 2013;41:711-8.

33. Lee HJ, Lee YJ, Choi CW, et al. Rosiglitazone, a peroxisome proliferatoractivated receptor- $\gamma$ agonist, restores alveolar and pulmonary vascular development in a rat model of bronchopulmonary dysplasia. Yonsei Med J 2014;55:99-106.

This work is licensed under a Creative Commons Attribution-NonCommercial-ShareAlike $\quad \mathbf{4 . 0}$ International License. The images or other third party material in this article are included in the article's Creative Commons license, unless indicated otherwise in the credit line; if the material is not included under the Creative Commons license, users will need to obtain permission from the license holder to reproduce the material. To view a copy of this license, visit http://creativecommons.org/ licenses/by-nc-sa/4.0/ 